\title{
11 Hypogonadismus
}

\author{
Frank-Michael Köhn
}

\subsection{Definition}

Der Hypogonadismus des Mannes ist gewöhnlich definiert als endokrine Funktionsstörung der Hoden und führt daher zu einem Testosteronmangel. Teilweise wird der Begriff des Hypogonadismus auch weiter gefasst und schließt dann Störungen der Spermatogenese mit reduzierter Spermienzahl und die eingeschränkte Produktion nicht-steroidaler Hormone wie das aus den Leydig-Zellen stammende Inhibin mit ein (Jockenhövel u. Schubert 2008). Der vorliegende Beitrag fokussiert auf die Ursachen, Klinik und Behandlung des Testosteronmangels des Mannes.

\subsection{Physiologische Grundlagen}

Die Produktion des Testosterons unterliegt einem Regelkreislauf, in den Hypothalamus, Hypophyse und Leydig-Zellen eingebunden sind. Gonadotrop-infreisetzendes Hormon (GnRH) wird pulsatil ca. alle go bis 120 Minuten aus dem Hypothalamus freigesetzt. Über das Pfortadersystem gelangt dieses Dekapeptid zum Hypophysenvorderlappen, wo es nach Bindung an spezifische Rezeptoren der Zielzellen die Produktion und Sekretion der Gonadotropine Follikel-stimulierendes Hormon (FSH) und Luteinisierendes Hormon (LH) bewirkt. Jedem CnRH-Puls folgt ein LH-Puls. LH gelangt über den Blutkreislauf zu den Leydig-Zellen des Hodens, bindet dort an einen spezifischen Rezeptor und stimuliert so die Produktion von Testosteron aus Cholesterin. Die Testosteronwerte im Serum weisen eine zirkadiane Rhythmik auf mit den höchsten Konzentrationen am Morgen und ca. 1/4 niedrigeren Werten am Abend.

Mehr als 95\% des im Körper zirkulierenden Testosterons werden im Hoden produziert (täglich 5-7 mg). Im Plasma wird Testosteron zu > 40\% an das sexualhormonbindende Globulin (SHBG) und zu > 50\% an Albumin gebunden. Nur ca. 2\% des Testosterons sind ungebunden (freies Testosteron). Im peripheren Gewebe wird Testosteron in den Zielzellen durch 5a-Reduktase zu dem biologisch aktiven 5a-Dihydrotestosteron (DHT) reduziert bzw. z.B. im Fettgewebe zu Östradiol aromatisiert. 
Beim Menschen werden zwei Isotypen der 5a-Reduktase unterschieden. Der Typ I (Chromosom 5) kommt in Haut und Leber, Typ II (Chromosom 2) in der Haut des Cenitale, den akzessorischen Geschlechtsdrüsen, Prostata und Haarfollikeln vor. Die Androgenwirkungen werden durch spezifische Androgenrezeptoren der Zielzellen vermittelt. Der Androgen-Rezeptor ist ein Steroid-Rezeptor. Die Zahl der CAG-repeats des kodierenden Gens bestimmt die Transkriptionsstärke des Rezeptors und damit die Androgenwirkung (Zitzmann 2009).

Androgenabhängige Organe bzw. Zellen sind die Haut (Haare, Talgdrüsen), Muskeln, Leber, Niere (Produktion von Erythropoetin), Knochen, Knochenmark (Blutstammzellen), Gehirn, Prostata und männliches Genitale.

Der Regelkreislauf zwischen Hypothalamus und Hypophyse wird durch Testosteron und seine Metabolite wesentlich reguliert. Testosteron und DHT hemmen die Freisetzung von FSH und LH über eine Beeinflussung der pulsatilen Sekretion des CnRH (Köhn 2004).

Die Testosteronproduktion unterliegt einem Regelkreislauf mit Beteiligung von Hypothalamus, Hypophyse und Gonaden.

\subsection{Klassifizierungen des Hypogonadismus}

Klinische Symptome, diagnostische Abklärung und Therapie des Hypogonadismus des Mannes hängen wesentlich von der Ursache und dem Zeitpunkt seines Auftretens ab. Der Aspekt eines eventuell aktuellen oder zukünftigen Kinderwunsches muss heute grundsätzlich Berücksichtigung bei der Beratung hypogonadaler Männer finden (s. Kap. II.1). So ist eine hochdosierte Testosteronsubstitution bei Männern mit Hypogonadismus und Kinderwunsch in der Regel kontraindiziert, da durch Suppression der Gonadotropine LH und FSH die Spermatogenese unterdrückt wird. Auch vor neueren Methoden der assistierten Reproduktion wie z.B. der testikulären Spermienextraktion nach Hodenbiopsien bei Patienten mit nicht-obstruktiver Azoospermie und Hypogonadismus sollte eine hochdosierte Substitution mit Testosteron zunächst unterbleiben; es kann allenfalls eine Induktion der körpereigenen Testosteronproduktion durch Gabe von humanem Choriongonadotropin (hCG) oder eventuell auch Antiöstrogenen erfolgen.

Klinisch sinnvolle Einteilungen des Hypogonadismus betreffen die anatomische Lokalisation der Schädigung im hypothalamo-hypophysären-gonadalen Regelkreislauf und genetisch bedingte oder erworbene Formen des Testosteronmangels (s. Tab. 1). Die klinischen Symptome werden in Abhängigkeit von der Ursache bedingt durch einen präpubertär oder postpubertär aufgetretenen Hypogonadismus.

Einen besonderen Stellenwert nimmt der sogenannte Altershypogonadismus ein, der international als „late-onset hypogonadism“ (LOH) bezeichnet wird.

\subsubsection{Primärer und sekundärer Hypogonadismus}

In Abhängigkeit vom Ort der Schädigung des endokrinologischen Regelkreises werden ein primärer und sekundärer Hypogonadismus differenziert. 
Tab. 1 Ursachen für Hypogonadismus

\begin{tabular}{ll} 
primärer Hypogonadismus & sekundärer Hypogonadismus \\
Anorchien (angeboren, erworben) & Tumore/Adenome/Metastasen \\
\hline Gonadendysgenesien & Medikamente \\
\hline Leydig-Zell-Aplasie & Trauma \\
\hline Enzymdefekte der Testosteronbiosynthese & Infektionen \\
\hline Klinefelter-Syndrom & Ischämie \\
\hline XYY-Syndrom & latrogen (Z.n. Operationen) \\
\hline XX-Mann-Syndrom & Hyperprolaktinämie \\
\hline Infektionen (Orchitis) & Idiopathischer hypogonadotroper Hypogonadismus \\
\hline Exogene Noxen (Chemotherapie, Radiatio) & Kallmann-Syndrom \\
\hline Allgemeinerkrankungen & Konstitutionelle Pubertas tarda \\
\cline { 2 - 2 } & Prader-Labhart-Willi-Syndrom \\
\cline { 2 - 2 } & Laurence-Moon-Bardet-Biedl-Syndrom
\end{tabular}

Beim primären Hypogonadismus ist die Produktion des Testosterons in den LeydigZellen des Hodens gestört. Die LH-Konzentrationen im Serum sind im Sinne einer Gegenregulation erhöht (hypergonadotroper Hypogonadismus). Typische Beispiele für einen primären Hypogonadismus sind das Klinefelter-Syndrom, Hodenfunktionsstörungen nach Hodenhochstand oder erworbene Schädigungen, z.B. nach Verletzungen oder infolge Chemotherapie.

Beim sekundären Hypogonadismus sind entweder die Sekretion von GnRH im Hypothalamus mit nachfolgender Stimulation der Hypophyse oder die Sekretion von LH durch einen Schaden in der Hypophyse beeinträchtigt, sodass die Stimulation der Leydig-Zellen ausbleibt. FSH und LH sind im Serum erniedrigt, und eine Stimulation des Hodens fehlt (hypogonadotroper Hypogonadismus). Eine hypophysäre Schädigung liegt z. B. bei Hypophysentumoren wie Prolaktinomen vor. Das Kallmann-Syndrom und der idiopathische hypogonadotrope Hypogonadismus dagegen sind typische Beispiele für einen sekundären Hypogonadismus durch eine Schädigung des Hypothalamus.

Diese Unterscheidung hat den Vorteil, dass sie Rückschlüsse auf die Therapie erlaubt. Beim primären Hypogonadismus ist in der Regel nur eine Substitution mit Testosteron möglich. Im Falle eines sekundären Hypogonadismus ist bei bestehendem Kinderwunsch eine Gabe von GnRH, hCG (mit LH-Wirkung) oder FSH sinnvoll. Besteht kein Kinderwunsch mehr, wird auch hier auf die kostengünstigere Testosteronsubstitution übergegangen.

Die Unterscheidung zwischen primärem und sekundärem Hypogonadismus hat wesentliche Bedeutung für die Therapie. 


\subsubsection{Genetisch bedingte Formen des Hypogonadismus}

Es gibt verschiedene Formen des genetisch bedingten Hypogonadismus mit z.T. sehr seltenen Syndromen. In der klinisch-andrologischen Routine haben das KlinefelterSyndrom und das Kallmann-Syndrom die größte Bedeutung.

\section{Klinefelter-Syndrom}

Das Klinefelter-Syndrom tritt mit einer Prävalenz von ca. 1:500 auf und ist durch den Karyotyp XXY, Mosaike verschiedener Zell-Linien (47,XXY/46,XY) oder mehrere X- oder Y-Chromosomen (z.B. 48, XXXY oder 48,XXYY) gekennzeichnet.

Nach manchmal verzögerter Pubertät kann sich ein eunuchoider Hochwuchs ausbilden, daneben andere Symptome von Androgenmangel (reduzierte Körperbehaarung, Osteoporose, beidseitige Gynäkomastie, geringe Libido); die Hoden sind verkleinert (<5 ml), FSH und LH erhöht (hypergonadotroper Hypogonadismus). Häufig fehlt aber der „typische“ klinische Habitus, der in den gängigen Lehrbüchern dargestellt wird, sodass die meisten Patienten mit Klinefelter-Syndrom äußerlich zunächst nicht erkannt werden.

Im Spermiogramm fallen meist ein fehlender Nachweis von Spermien (Azoospermie) und Verminderung der Ejakulatmenge (Bläschendrüseninsuffizienz bei Androgenmangel) auf. Die Spermatogenese kann teilweise erhalten sein. Auch bei Azoospermie können in Hodenbiopsien noch Inseln von Spermatogenese nachweisbar sein, aus denen Spermien für eine intrazytoplasmatische Spermieninjektion gewonnen werden können. Unter Berücksichtigung der publizierten Fälle kann davon ausgegangen werden, dass testikuläre Spermatozoen in 20-69\% der Patienten gefunden werden (Schiff et al. 2005; Ramasamy et al. 2009).

Die Angaben über die mit testikulären Spermatozoen von Männern mit KlinefelterSyndrom erreichten Schwangerschaften schwanken; es werden sowohl signifikant niedrigere Schwangerschaftsraten als auch vergleichbare Ergebnisse wie mit Spermien von Männern mit nicht-obstruktiver Azoospermie anderer Genese berichtet. Ein erhöhtes Risiko für die Nachkommen von Männern mit Klinefelter-Syndrom nach Methoden der assistierten Reproduktion scheint nicht zu bestehen (Fullerton et al. 2010).

\section{Kallmann-Syndrom}

Beim Kallmann-Syndrom und Idiopathischen Hypogonadotropen Hypogonadismus (IHH) führt eine gestörte Sekretion von $\mathrm{GnRH}$ zu einer ausbleibenden Stimulation der Hypophyse mit niedrigen Serum-Spiegeln von FSH und LH. Dadurch erfolgt auch keine Stimulation der Spermatogenese und Testosteronproduktion im Hoden. Es handelt sich um relativ seltene Erkrankungen. Bei Männern treten sie mit einer Häufigkeit von 1:8.00o bis 1:10.000 auf.

Beide Erkrankungskomplexe können vererbbar sein. Dem Kallmann-Syndrom und dem IHH liegt aber nicht nur ein definierter Gendefekt zugrunde. Defekte Gene wurden auf dem X-Chromosom (KAL-Gen) und dem Chromosom 8 identifiziert. Die Erkrankung kann autosomal-dominant, autosomal-rezessiv und X-chromosomal-rezessiv vererbt werden. In den meisten Fällen haben die betroffenen Patienten die 
endokrine Störung aber nicht von ihren Eltern vererbt bekommen, sondern die Erkrankung ist spontan aufgetreten.

Die zur Diagnose führenden Symptome sind klinische Zeichen eines Hypogonadismus und eine Riechstörung (beim Kallmann-Syndrom).

Die Klinik zeigt eine große Variabilität. In der Regel werden die Patienten zunächst wegen einer ausbleibenden oder nur gering ausgeprägten Pubertät vorgestellt. Die Hodengröße liegt unter $5 \mathrm{ml}$, der Penis ist unterentwickelt, die sekundäre Behaarung (Bartwuchs, Pubesbehaarung, Axillarbehaarung) fehlt oder ist nur ansatzweise vorhanden, das Fettverteilungsmuster ist weiblich.

IHH und Kallmann-Syndrom können sich aber auch erst im Erwachsenenalter manifestieren. Die Patienten werden dann meist zur Abklärung einer Infertilität oder eines Testosteronmangels mit entsprechender klinischer Symptomatik einschließlich Abnahme der Libido oder Auftreten von Erektionsstörungen vorstellig. Die Riechstörung wird von den Männern meist nicht spontan angesprochen, sondern muss gezielt erfragt werden (Köhn 2004).

\subsection{Klinik und Anamnese bei Hypogonadismus}

Die Klinik bei erniedrigten Testosteronwerten im Serum wird im Wesentlichen durch den Zeitpunkt der Manifestation des Hypogonadismus bestimmt (Köhn 2004) (s. Tab. 2). Pubertas tarda, eunuchoider Hochwuchs und geringe Ausprägung der sekundären Geschlechtsmerkmale deuten auf einen schon präpubertär entstandenen Hypogonadismus hin. Für die regelrechte Entwicklung der männlichen Geschlechtsorgane und deren Funktion ist die Anwesenheit der Androgene bereits intrauterin bis zum Ende der Pubertät obligat.

Tab. 2 Klinische Symptome des Hypogonadismus (nach Jockenhövel u. Schubert 2008)

\begin{tabular}{lll} 
& präpubertär & postpubertär \\
\hline Knochen & eunuchoidale Proportionen, Osteoporose & Osteoporose \\
\cline { 2 - 3 } Kehlkopf & kein Stimmbruch & unverändert \\
Sekundäre & horizontale Begrenzung der Pubesbehaarung, & Abnahme der Haardichte \\
\hline Körperbehaarung & kein Bartwuchs, keine androgenetische Alopezie & \\
\hline Haut & keine Seborrhoe, keine Akne & Atrophie, Blassheit \\
\hline Knochenmark & Anämie & Anämie \\
\hline Muskeln & unterentwickelt & Abnahme der Muskelmasse \\
\hline Penis & infantil & keine Größenänderung \\
\hline Spermatogenese & fehlt & Einschränkung \\
\hline Prostata & unterentwickelt & Größenabnahme \\
\hline Ejakulat & Anejakulation, geringes Volumen & Abnahme des Volumens \\
\hline Libido & fehlt & Abnahme \\
\hline Potenz & fehlt & Abnahme \\
\hline
\end{tabular}


Androgene haben somatische (Körpermuskulatur, Fettverteilung, Knochendichte, Hämatopoese, Spermatogenese, Körperbehaarung und Funktionen von Schwellkörpergewebe, Bläschendrüsen, Prostata und Nebenhoden) und psychotrope Effekte (Libido, Wohlbefinden, Leistungsfähigkeit und Stimmung).

Subjektive Symptome des Testosteronmangels sind daher Abnahme der Libido, Erektionsstörungen, depressive Stimmungslage, Abnahme der allgemeinen Aktivität, Lustlosigkeit, Hitzewallungen und Nachlassen der Muskelkraft. Sekundäre Körperbehaarung und Rasurfrequenz können vermindert sein. Früh in der Kindheit diagnostizierte pathologische Befunde wie Hodenhochstand oder Mumpsorchitis müssen erfasst werden.

Insbesondere bei älteren Männern sollte auch immer gezielt nach begleitenden chronischen Erkrankungen gefragt werden, die mit Hypogonadismus assoziiert sein können (Diabetes mellitus, Niereninsuffizienz, Lebererkrankungen, Asthma bronchiale, chronisch-entzündliche Darmerkrankungen, chronische Infektionen wie z.B. HIV, Tuberkulose oder Lepra, maligne Erkrankungen). Medikamenteneinnahme wie z.B. chronische Anwendung von Kortikosteroiden können ebenso wie Ketokonazol, Digitalis, Cimetidin, Spironolacton oder Flutamid das Testosteron im Serum vermindern oder seine Wirkung reduzieren. Ähnliche Wirkungen haben Genussgifte wie Alkohol oder Drogen wie z.B. Marihuana, Opiate und Kokain, die die Sekretion von GnRH negativ beeinflussen. Die Anamnese sollte auch andere potenziell schädigende Faktoren wie Operationen, Traumen erfassen und seltenere Symptome wie z.B. Sehstörungen bei hypophysären Tumoren oder pathologische Frakturen mitberücksichtigen (Köhn 2004).

Die klinischen Symptome korrelieren teilweise mit den Konzentrationen des Testosterons im Serum. Libidoverlust und Antriebsschwäche können dabei schon bei niedrig-normalen Gesamttestosteronwerten zwischen 12 und $15 \mathrm{nmol} / \mathrm{l}$ beobachtet werden. Depressive Störungen, Schlafstörungen und Konzentrationsschwäche treten signifikant häufiger bei niedrigeren Werten zwischen 8 und $10 \mathrm{nmol} / \mathrm{l}$ auf, während eine erektile Dysfunktion oder Hitzewallungen erst bei noch niedrigeren Gesamttestosteronwerten im Serum unter $8 \mathrm{nmol} / \mathrm{l}$ gehäuft auffallen (Zitzmann et al. 2006).

\subsection{Diagnostik bei Hypogonadismus}

\subsubsection{Endrokrinologische Basisdiagnostik}

Bei Verdacht auf einen Hypogonadismus sollten zunächst Testosteron (Grenzbereich: 10-12 nmol/l, Normbereich: 12-35 nmol/l), LH (Normbereich: 1-8 mIU/ml) und FSH (Normbereich: 1-10 $\mathrm{mIU} / \mathrm{ml}$ ) bestimmt werden. Hierbei ist zu beachten, dass diese Werte in Abhängigkeit vom verwendeten Bestimmungsverfahren variieren können. Zudem müssen Besonderheiten bei der Freisetzung der Hormone berücksichtigt werden. Da die Testosteronkonzentrationen im Serum zirkadiane Schwankungen aufweisen, sollten Bestimmungen dieses Hormons bevorzugt am Morgen zu ähnlichen Zeiten erfolgen.

Durch Bestimmung des SHBG kann der freie Androgen-Index ermittelt werden.

Die pulsatile Freisetzung der Gonadotropine muss berücksichtigt werden; so empfiehlt sich eine Wiederholung der Bestimmung bei unerklärlich hohen oder niedri- 
gen Werten von LH oder FSH, da eine Blutabnahme exakt zum Zeitpunkt eines Pulses oder unmittelbar vor dem nächsten Puls erfolgt sein könnte. Die LH- bzw. FSH-Werte erlauben erst die Unterscheidung in hypogonadotropen oder hypergonadotropen Hypogonadismus und damit die Differenzierung zwischen testikulären oder hypothalamisch-hypophysären Ursachen des Hypogonadismus.

Prolaktin sollte bei erniedrigten Testosteronwerten im Serum sowie bei Verdacht auf hypophysäre Prozesse oder bei entsprechender Klinik (z.B. Gynäkomastie) bestimmt werden. Wenn Prolaktin bestimmt wird, sollte dies frühestens 2 Stunden nach dem Aufwachen (nächtliche Prolaktinerhöhungen) und nicht nach üppigen Mahlzeiten oder körperlichen Anstrengungen (auch Geschlechtsverkehr!) erfolgen. Ist das Prolaktin im Serum erhöht, sollten zunächst eine zweite Untersuchung abgewartet und weitere Einflussfaktoren oder assoziierte Erkrankungen wie z.B. Einnahme von Medikamenten (Tranquilizer) oder Hypothyreose ausgeschlossen werden (Köhn 2004).

Testosteronbestimmungen im Serum erfolgen am Vormittag zwischen 8:00 und 11:00 Uhr.

\subsubsection{Endokrinologische Funktionsdiagnostik}

In der Praxis wird vor allem der GnRH-Test als Funktionstest angewendet. Er wird bei reduzierten oder niedrig-normalen Konzentrationen der Gonadotropine durchgeführt und dient der Unterscheidung zwischen hypothalamisch oder hypophysär bedingten verminderten Gonadotropin-Konzentrationen im Serum. Steigen die Gonadotropine nach Injektion von $\mathrm{GnRH}$ an, liegt ein hypothalamischer Schaden vor; bleibt der Anstieg der Gonadotropin-Konzentrationen im Serum nach Injektion von $\mathrm{GnRH}$ aus, ist die Störung in der Hypophyse lokalisiert. Beim GnRH-Test erfolgt die Bestimmung der Gonadotropine 30 Minuten nach Injektion von $\mathrm{GnRH}$.

Mit dem hCG-Test kann die Funktion der Leydig-Zellen und somit die endokrine Reservekapazität des Hodens überprüft werden. Seine klinische Aussagekraft bei der weiteren Abklärung eines Hypogonadismus ist aber mit Ausnahme von Sonderindikationen (z.B. Anorchie, Pubertas tarda) eingeschränkt.

\subsubsection{Sonstige Untersuchungen}

Zusätzlich zu den endokrinologischen Untersuchungen können bei jüngeren Patienten mit Hypogonadismus Ejakulatuntersuchungen notwendig werden. Bei gesicherter Diagnose empfiehlt sich auch eine Knochendichtebestimmung als Ausgangswert. Sind die betroffenen Patienten älter, muss besondere Sorgfalt auf Untersuchungen der Prostata verwendet werden (Palpation, Sonographie, PSA-Wert), da bei der Behandlung des Hypogonadismus mit Testosteronpräparaten die Beschleunigung des Wachstums eines bereits bestehenden Prostatakarzinoms nicht ausgeschlossen werden kann.

Weitere Blutuntersuchungen nach gesichertem Hypogonadismus beinhalten vor allem ein Blutbild (Anämie?) und vor Beginn der Substitutionstherapie Bestimmungen der Blutfette. 
Besondere bildgebende Verfahren (CT/MRT) und molekularbiologische Spezialuntersuchungen mit Bestimmung von Deletionen im Androgen-Rezeptor-Gen, LH- und FSH-Gen und Untersuchung von CAG-Repeats sind speziellen Indikationen und klinischen Bildern vorbehalten.

\subsection{Therapie des Hypogonadismus}

Die genaue Diagnostik und Klassifikation eines Testosteronmangels hat wesentlichen Einfluss auf die Therapie. Ein hypogonadotroper Hypogonadismus kann in Abhängigkeit vom Ort der Schädigung des Regelkreislaufes durch GnRH (GnRH-Pumpe bei hypothalamischen Schäden) oder hCG (mit LH-Aktivität) und reines bzw. rekombinant hergestelltes FSH therapiert werden. Zusätzlich muss bei diesen Formen des Hypogonadismus berücksichtigt werden, ob ein aktueller Kinderwunsch besteht oder nicht. Bei aktuellem Kinderwunsch sollte in Fällen eines sekundären Hypogonadismus der Testosteronmangel immer durch eine Stimulation der Leydigzellen durch hCG behoben werden. Nach ca. sechs Wochen kann dann zusätzlich reines oder rekombinant hergestelltes FSH gegeben werden, da die alleinige Gabe von Testosteron in diesen Fällen die Spermatogenese nicht aktivieren könnte (s. Kap. II.1).

Nach Beendigung des Kinderwunsches wird in der Regel eine Testosteronsubstitutionstherapie durchgeführt, da diese kostengünstiger und einfacher durchzuführen ist als eine Gabe von hCG und FSH.

Vor einer Testosteronsubstitutionstherapie sollte nach aktuellem Kinderwunsch gefragt werden.

\subsubsection{Therapie des sekundären Hypogonadismus bei Kinderwunsch}

\section{Therapie mit GnRH}

GnRH stimuliert die Freisetzung von LH und FSH aus der Hypophyse; es steht als synthetisches Produkt zur Verfügung (Gonadorelin). Die physiologisch pulsatile Sekretion von GnRH wird durch eine Pumpe imitiert, die Gonadorelin pulsatorisch subkutan injiziert. Indikationen: sekundärer hypogonadotroper Hypogonadismus, $\mathrm{Pu}-$ bertas tarda. Dosierung: 5-20 $\mu \mathrm{g}$ Gonadorelin pro Puls alle 120 Minuten, mehrere Monate. Unter dieser Therapie normalisieren sich die T-Spiegel; es kommt zur Zunahme des Hodenvolumens (Büchter et al. 1998).

\section{Therapie mit hCG und FSH}

hCG ist ein Polypeptid, das analog dem LH die T-Produktion in den Leydigzellen stimuliert. Es ist zur Behandlung des Hodenhochstandes, der Pubertas tarda und, bei Erwachsenen, zur Therapie des hypogonadotropen Hypogonadismus zugelassen (2-3 x/Woche 1.0oo-2.500 IE i.m., s.c.). Unter dieser Therapie normalisieren sich die T-Spiegel, das Hodenvolumen nimmt zu. Die Substitution mit hCG wird bei gleichzeitig bestehendem Kinderwunsch mit rekombinatem FSH oder humanem Menopausengonadotropin kombiniert (Büchter et al. 1998). 
Hochgereinigtes und rekombinantes FSH stimulieren die Spermatogenese. Bei hypogonadotropem Hypogonadismus wird FSH (3 x/Woche 75-150 IE i.m., s.c.) zusammen mit hCG appliziert. Bei längeren Behandlungspausen, oder wenn der Patient zuvor mit Testosteron substituiert wurde, empfiehlt sich zunächst die Gabe von hCG zur Stimulation der körpereigenen Testosteron-Produktion; nach 1-3 Monaten wird dann zusätzlich FSH gegeben. In bis zu 90\% setzt die Spermatogenese wieder ein.

\section{Substitution mit Testosteron}

Für die Testosteronsubstitution stehen verschiedene Applikationswege zur Verfügung. Die am häufigsten verwendeten sind die intramuskuläre und transdermale Applikationsform. Seltener finden orale, bukkale oder subkutane Substitutionen von Testosteron Anwendung.

\section{Intramuskuläre Gabe von Testosteronestern}

Testosteronenantat ist eine veresterte Form des körpereigenen Testosterons. Es ist beim Mann zur Behandlung des Hypogonadismus, der Pubertas tarda, des überschießenden Längenwachstums von Knaben sowie der Therapie aplastischer und renaler Anämien zugelassen.

Die Veresterung erfolgt, um die Absorption nach intramuskulärer Injektion zu verlangsamen und so die Verfügbarkeit des Hormons nach einmaliger Injektion über einen längeren Zeitraum sicherzustellen. Mögliche Nebenwirkungen beim Mann sind: Gynäkomastie, Ödeme, Priapismus, Ödemneigung, Akne, Gynäkomastie, Hemmung der Spermatogenese, Hyperkalzämie, Unverträglichkeitsreaktionen. Wird Testosteronenantat zur Substitutionstherapie bei Hypogonadismus eingesetzt, erfolgen die Injektionen in zwei- bis vierwöchigen Abständen (Einzeldosis $250 \mathrm{mg}$ Testosteronenantat). Der Nachteil dieser Therapieform besteht darin, dass zunächst über mehrere Tage nach der Injektion supraphysiologische Testosteronkonzentrationen erreicht werden.

Daher ist Testosteronenantat weitgehend durch die Applikation von Testosteronundekanoat $(1.000 \mathrm{mg})$ in öliger Lösung abgelöst worden. Hierbei erfolgen die ersten beiden Injektionen im Abstand von 6 Wochen; dann werden die Injektionen alle drei Monate vorgenommen. Hiermit lassen sich stabile Testosteronwerte erzielen. Da die Injektionsmenge $4 \mathrm{ml}$ ist, sollte die Injektion langsam und im Liegen erfolgen. Supraphysiologische Spiegel von Serum-Testosteron werden bei ordnungsgemäßer Anwendung nur selten beobachtet.

\section{Transdermale Applikation von Testosteron}

Bei den in Deutschland zugelassenen testosteronhaltigen Gelen wird ein Wasser/Alkohol-Gemisch verwendet, um Testosteron in Lösung zu bringen und dessen Aufnahme durch die Haut zu verbessern. Das Gel wird dünn und ohne einzureiben bevorzugt am Morgen auf die unversehrte Haut an Armen, Schultern oder Bauch aufgetragen. Dazu stehen Beutel mit verschiedenen Testosteronmengen (25 bis $125 \mathrm{mg}$ ) zur Verfügung. Die Standarddosis beträgt in der Regel $5 \mathrm{mg}$.

Bevor sich der Patient ankleidet, sollte ca. 5 Minuten gewartet werden. Die Hände müssen mit Wasser und Seife gereinigt werden, um dort Gelreste zu entfernen. Di- 
rekter Körperkontakt mit anderen Personen (v.a. Frauen und Kinder) sollte innerhalb der ersten 6 Stunden nach dem Auftragen vermieden werden. Andernfalls sollte zuvor gründlich geduscht werden. Von dem im Gel enthaltenen Testosteron werden ca. 10\% in den Körper aufgenommen. Durch die Anwendung des Gels am Morgen werden physiologische Konzentrationen des Testosterons im Serum bereits nach wenigen Tagen erreicht. Dann sollten Kontrollen der Testosteronwerte im Blut vor der nächsten Anwendung erfolgen. Da das Gel hochprozentigen Alkohol enthält, sollte es zur Vermeidung von Hautreizungen nicht auf die Genitalhaut aufgetragen werden.

\section{Orale Applikation von Testosteronestern}

Testosteronundecanoat ist ein in Position $17 \beta$ mit Undecansäure verestertes Testosteron. Dadurch wird die Substanz so lipophil, dass sie aus dem Gastrointestinalsystem in die Lymphbahn aufgenommen wird. Vom Ductus thoracicus gelangt Testosteronundecanoat über die Vena subclavia in den Blutkreislauf und schließlich zu den Zielorganen. Auf diese Weise wird eine vorzeitige Metabolisierung des Testosterons in der Leber durch den „first pass“-Effekt vermieden. Nach oraler Aufnahme werden die höchsten Plasmakonzentrationen von Testosteronundecanoat im Durchschnitt nach 4 Stunden gemessen, wobei Einnahme mit fettreicher Mahlzeit die Absorption noch erhöht. Zur Substitutionstherapie werden 120-160 mg Testosteronundecanoat in Form von Kapseln (mit Castoröl) täglich verordnet.

Wegen unzuverlässiger Resorption aus dem Gastrointestinaltrakt hat sich diese Form der Testosteronsubstitution aber nicht durchgesetzt.

\subsubsection{Kontraindikationen der Testosteronsubstitution}

Eine Substitution mit Testosteron ist kontraindiziert bei Männern mit Prostata- oder Mammakarzinom. Vorsicht ist bei schweren Leber-, Nieren- oder Herzerkrankungen (Ödemneigung) oder Bluthochdruck (weiterer Anstieg) geboten.

Weiterhin kann die Androgenbehandlung zu einer Anhebung des Hämoglobin- und Hämatokritwertes führen sowie eine Schlafapnoeneigung verstärken. Somit bestehen weitere Kontraindikationen für Erkrankungen wie Polyzythämia, chronisch obstruktive Lungenerkrankungen und eine bekannte Schlafapnoe.

Durch eine Testosteronsubstitution bei Hypogonadismus wird eine vorher verkleinerte Prostata auf ihr Normalvolumen vergrößert und dadurch auch der PSA-Wert in den Normbereich angehoben.

Von großer Bedeutung ist hingegen die Problematik des Prostatakarzinoms bei älteren Patienten. Liegt ein solches in einem klinischen Stadium vor, ist es nicht ausgeschlossen, dass durch eine externe Testosterongabe ein Wachstum und somit eine Metastasierung stimuliert werden können. Hieraus resultiert, dass vor und unter jeder Androgenbehandlung ein Prostatakarzinom mittels rektaler Untersuchung oder transrektaler Prostatasonographie und Bestimmung des PSA im Serum ausgeschlossen werden muss. Nach erfolgreich und radikal behandeltem Prostatakarzinom besteht entsprechend der aktuellen Leitlinien bei Beachtung der Sicherheitsparameter keine Kontraindikation mehr für die Behandlung von älteren Männern mit Hypogonadismus (Wang et al. 2009). 
Bei Jugendlichen mit noch nicht abgeschlossenem Längenwachstum führt eine gesteigerte Testosteronzufuhr zu einem verfrühten Verschluss der Epiphysenfugen und kann somit einen Minderwuchs verursachen.

\section{Vor Beginn der Testosteronsubstitutionstherapie des älteren Mannes muss ein Prostatakarzinom ausgeschlossen werden.}

\subsubsection{Sicherheitsparameter unter Testosteronsubstitutionstherapie}

Vor Beginn der Testosterontherapie sollten neben den endokrinologischen Untersuchungen Bestimmungen der Leberwerte, der Fettwerte (Gesamt-Cholesterin, LDLCholesterin, HDL-Cholesterin), des Blutbildes sowie bei älteren Männern (> 50 Jahre) des PSA erfolgen. Zusätzlich sind eine Prostatapalpation und transrektale Prostatasonographie sowie eine Messung der Knochendichte notwendig (Jockenhövel u. Schubert 2004).

Nach drei Monaten werden dann neben der Erfassung anamnestischer Angaben zu Symptomen des Testosteronmangels und Untersuchungen testosteronabhängiger Organe Kontrollen von Testosteron, LH, Blutbild, Leberwerten, Fettwerten sowie bei älteren Männern PSA erfasst. Testosteron sollte sich normalisieren und das prätherapeutisch eventuell erhöhte LH absinken. Weitere Kontrollen der oben genannten Parameter erfolgen nach 6 und 12 Monaten. Eine Überprüfung der Knochendichte ist frühestens nach einem Jahr sinnvoll.

\subsection{4 „Late onset hypogonadism“ (LOH)}

Die mittleren Testosteronkonzentrationen nehmen zwischen dem 4. und 7. Lebensjahrzehnt um ca. $1 \%$ pro Jahr ab, bleiben aber dennoch häufig innerhalb der für gesunde Männer geltenden Normwerte. Nur ca. 20\% der 6o-80-jährigen Männer haben einen echten Hypogonadismus mit einem Serum-Testosteronspiegel unter $12 \mathrm{nmol} / \mathrm{l}$ (Gooren 1996; Kaufman u. T’Sjoen 2002).

Hierbei ist aber zu berücksichtigen, dass zwischen 10-12 nmol/l ein Graubereich liegt und ein erniedrigter Testosteronwert bei Fehlen subjektiver oder objektivierbarer Beschwerden noch keine unbedingte Therapieindikation darstellt. Eine unkritische Einnahme oder Verschreibung von Testosteron bei älteren Männern ist demnach ohne vorhergehende endokrinologische Abklärung abzulehnen (s. Kap. II.13).

In der Regel wird eine Testosteronsubstitution aber bei einem Gesamttestosteronwert von unter $8 \mathrm{nmol} / \mathrm{l}$ notwendig, wobei die Anwendung kürzer wirksamer Testosteronprodukte (z.B. Gele) gegenüber Depotpräparaten bevorzugt wird (Wang et al. 2009). Die Therapie hat positive Effekte auf die Stimmung, Wohlbefinden, Libido, Muskelstärke, Knochendichte, fettfreie Körpermasse, Erythropoese, kognitive Funktionen und kardiovaskuläre Risikofaktoren (Gooren 2010) (s. Tab. 3).

Eine internistische Betreuung ist häufig sinnvoll, da der Hypogonadismus des älteren Mannes mit einem metabolischen Syndrom (Übergewicht, erhöhte Insulinresistenz, Hypertonus, Fettstoffwechselstörungen) assoziiert sein kann. 


\section{Tab. 3 Effekte einer Testosterontherapie bei älteren Männern}

\begin{tabular}{lcc} 
& Alter & Testosteron \\
\hline Muskelmasse & $\downarrow$ & $\uparrow$ \\
\hline Muskelkraft & $\downarrow$ & $\uparrow$ \\
\hline Fettmasse & $\uparrow$ & $\downarrow$ \\
\hline Insulinresistenz & $\uparrow$ & $\downarrow$ \\
\hline diastolischer Blutdruck & $\uparrow$ & - \\
\hline kardiovaskuläre Mortalität & $\uparrow$ & $\uparrow$ \\
\hline Knochenmasse & $\downarrow$ & $?$ \\
\hline Frakturen & $\uparrow$ & $\uparrow$ \\
\hline Libido & $\downarrow$ & $\uparrow$ \\
\hline erektile Dysfunktion & $\uparrow$ & (besseres Ansprechen auf Phosphodiesterase-5-Inhibitoren) \\
\hline psychisches Wohlbefinden & $\downarrow$ & $\uparrow$ \\
\hline kognitive Funktionen & $\downarrow$ & $?-\uparrow$ \\
\hline
\end{tabular}

\section{Literatur}

Büchter D, Behre HM, Kliesch S, Nieschlag E (1998) Pulsatile GnRH or human chorionic gonadotropin/human menopausal gonadotropin as effective treatment for men with hypogonadotropic hypogonadism: a review of 42 cases. Eur I Endocrinol 139, 298-303

Fullerton G, Hamilton M, Maheshwari A (2010) Should non-mosaic Klinefelter syndrome men be labelled as infertile in 2009? Hum Reprod 25, 588-597

Gooren LIG (1996) The age-related decline of androgen in men: clinically significant? Brit J Urol 78, 763-768

Gooren LJG (2010) Androgens and male aging: Current evidence of safety and efficacy. Asian I Androl 12, 136-151 Jockenhövel F, Schubert M (2008) Männlicher Hypogonadismus. 3. Auflage, UNI-MED Verlag, Bremen

Kaufman JM, T'Sjoen G (2002) The effects of testosterone deficiency on male sexual function. Aging Male 5, 242-247

Köhn FM (2004) Diagnostik und Therapie des Hypogonadismus bei erwachsenen Männern. Hautarzt 55, 877-897

Ramasamy R, Ricci JA, Palermo GD, Gosden LV, Rosenwaks Z, Schlegel PN (2009) Successful fertility treatment for Klinefelter's syndrome. J Urol 182, 1108-1113

Schiff JD, Palermo GD, Veeck LL, Goldstein M, Rosenwaks Z, Schlegel PN (2005) Success of testicular sperm extraction and intracytoplasmic sperm injection in men with Klinefelter syndrome. I Clin Endocrinol Metab 90, 6263-6267

Wang C, Nieschlag E, Swerdloff RS, Behre H, Hellstrom W), Gooren L), Kaufman |M, Legros II, Lunenfeld B, Morales A, Morley JE, Schulman C, Thompson IM, Weidner W, Wu FC (2009) ISA, ISSAM, EAU, EAA and ASA recommendations: investigation, treatment and monitoring of late-onset hypogonadism in males. Aging Male 12, 5-12

Zitzmann M (2009) The role of the CAG repeat androgen receptor polymorphism in andrology. Front Horm Res 37 , $52-61$

Zitzmann M, Faber S, Nieschlag E (2006) Association of specific symptoms and metabolic risks with serum testosterone in older men. I Clin Endocrinol Metab 91, 4335-4343 